\title{
Beamline operating software B4 and automated crystallography suite at the Berkeley Center for Structural Biology
}

\author{
Kevin Royal ${ }^{a}$, John Taylor ${ }^{a}$, Randall Cayford ${ }^{a}$, Anthony Rozales ${ }^{a}$, Jeff Dickert ${ }^{a}$, Daniil \\ Prigozhin $^{\mathrm{a}}$, Jay Nix ${ }^{\mathrm{b}}$, Corie Ralston ${ }^{\mathrm{a}}$ and Marc Allaire ${ }^{\mathrm{a}}$ \\ ${ }^{a} B e r k e l e y$ Center for Structural Biology, Molecular Biophysics and Integrated Bioimaging, \\ Lawrence Berkeley National Laboratory, One Cyclotron Road, Berkeley, California 94720, USA \\ aMolecular Biology Consortium, One Cyclotron Road, Berkeley, California 94720, USA \\ Email Contact: kroyal@Ibl.gov
}

The Berkeley Center for Structural Biology has released "B4" Beamline Operating Software. The software suite provides tools and features that automate repetitive, tedious collection tasks that are prone to human error. New automated task management, crystal centering, rastering, and indexing tools can be used individually or combined for assisted or completely automated sample collection. B4 takes full advantage of recent hardware upgrades at the Berkeley Center for Structural Biology. A shutterless Pilatus detector and new computing hardware have increased processing speeds allowing prompt updating to the B4 data tab and our web-based portal. The new B4 software and automated crystallography suite is capable of collecting a dataset every 6 minutes providing unrivaled throughput and flexibility for the crystallographic community. 